\title{
Collective Frequencies and Metastability in Networks of Limit-Cycle Oscillators with Time Delay
}

\author{
Ernst Niebur, Heinz G. Schuster, ${ }^{(a)}$ and Daniel M. Kammen ${ }^{(b)}$ \\ Computation and Neural Systems Program, California Institute of Technology, Pasadena, California 91125
}

(Received 20 March 1991)

\begin{abstract}
We analyze the dynamic behavior of large two-dimensional systems of limit-cycle oscillators with random intrinsic frequencies that interact via time-delayed nearest-neighbor coupling. We find that even small delay times lead to a novel form of frequency depression where the system decays to stable states which oscillate at a delay and interaction-dependent reduced collective frequency. For greater delay or tighter coupling between oscillators we find metastable synchronized states that we describe analytically and numerically.
\end{abstract}

PACS numbers: $05.45 .+\mathrm{b}, 87.10 .+\mathrm{e}$

Arrays of coupled limit-cycle oscillators are of fundamental interest in physics [1-3], biology [4-8], and engineering [9]. In each of these cases, finite transmission velocities or discrete events, such as the propagation of information through a network node or "synapse," introduce delays in the system that are not commonly incorporated into the picture of interacting oscillators. The recognition that delay increases the dimensionality, and hence the complexity, of the system has focused efforts on those domains where delay is not a major factor, or where the system becomes chaotic [10-14]. The ubiquitous nature of time delay leads naturally to the subject of this Letter: an exploration of the dynamic behavior and metastable states of coupled time-delayed oscillators.

We study a system of $N$ coupled oscillators with phases $\phi_{i} \in[0,2 \pi]$. The Langevin dynamics of the system are described by $N$ differential-difference equations:

$$
\frac{d \phi_{i}(t)}{d t}=\omega_{0}+K \sum_{j} \sin \left[\phi_{j}(t-\tau)-\phi_{i}(t)\right]+\eta_{i}(t),
$$

where $K$ is the coupling constant, $\tau$ is the delay, and $\omega_{0}$ is the intrinsic frequency of the oscillators. The sum runs over all nearest neighbors of oscillator $i$. The temperature $T$ is incorporated in the usual way by the Gaussian noise term $\eta_{i}(t)$ :

$$
\begin{aligned}
& \left\langle\eta_{i}(t)\right\rangle=0, \\
& \left\langle\eta_{i}(t) \eta_{j}\left(t^{\prime}\right)\right\rangle=2 T \delta(i, j) \delta\left(t-t^{\prime}\right) .
\end{aligned}
$$

We simulated this system for $N=16384(128 \times 128)$ oscillators on the CM-2 Connection Machine, using a two-dimensional square lattice with periodic boundary conditions. We found the surprising result that even at low temperatures $\left(T \ll \omega_{0}\right)$ the system exhibits a strong "frequency suppression": With increasing coupling and delay the mean frequency, $\Omega=(1 / N) \sum_{i}\left\langle\dot{\phi}_{i}\right\rangle$ [the angular brackets denote the average over the random noise $\eta_{i}(t)$ ], of the system is drastically reduced as shown in Fig. 1 (as a function of $\tau$ ). We obtain a similar frequency depression if instead of a single frequency $\omega_{0}$ the oscillators have different intrinsic frequencies with a distribution that has a mean $\omega_{0}$ and a width comparable to $\omega_{0}$ (data not shown).

In order to understand this effect we consider a situation where all angles change with the same frequency $\Omega$ without fluctuation (i.e., $\phi_{i}=\Omega t+\alpha$ ) and obtain for $T=0$ from Eq. (1) the result

$$
\Omega=\omega_{0}-K n \sin (\Omega \tau),
$$

where $n$ is the number of neighbors (four in the case of a square lattice with nearest-neighbor interaction). This equation has multiple solutions. In Fig. 1 we plot the lowest stable frequency $\Omega_{\min } \approx \omega_{0} /(1+K n \tau)$ and obtain excellent agreement with our simulations.

It is to be expected that the approximation of common frequency and phase of all oscillators is less justified for intermediate values of the coupling $K$ than in the case of the strong coupling considered here, and that many-body effects will lead to more complicated phenomena. For the case we are interested in (strong coupling and small delays), however, the simple theory is obviously sufficient.

Two questions arise: (i) Why do we see only the small-

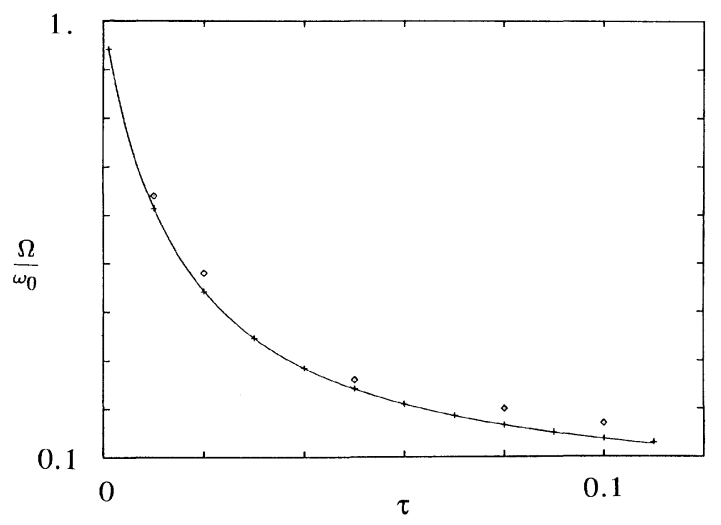

FIG. 1. Frequency suppression as a function of time delay $\tau$ (in units of $2 \omega_{0}^{-1}$ ) for two coupled oscillators (diamonds), for a two-dimensional array of 16384 oscillators (crosses), and the prediction from Eq. (2) (line). The average frequency $\Omega$ is plotted as a fraction of the intrinsic frequency $\omega_{0}$. The temperature is $T=10^{-4} \mathrm{~K}$. 


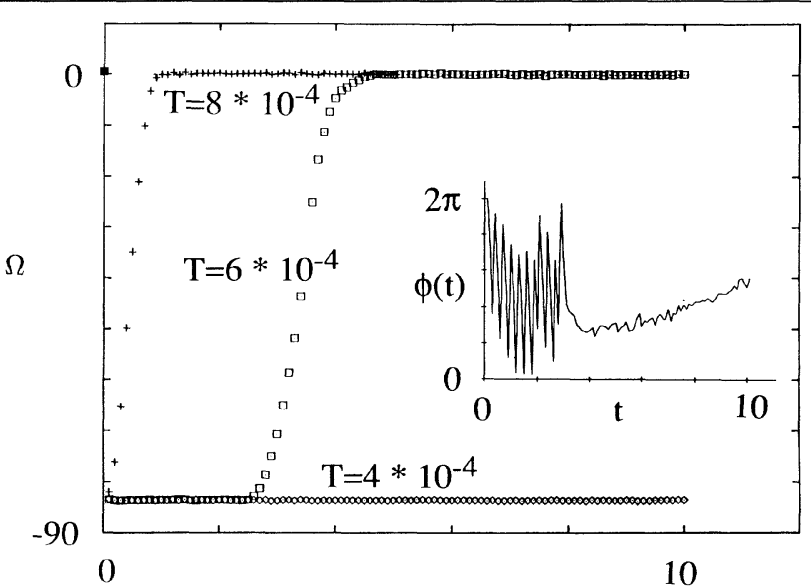

FIG. 2. Average frequency $\Omega$ for a system with initial conditions given in Eq. (3), as a function of time (in units of $2 \omega_{0}^{-1}$ ) for different temperatures $\left(T=4 \times 10^{-4} \mathrm{~K}\right.$, diamonds; $T=6$ $\times 10^{-4} \mathrm{~K}$, squares; $T=8 \times 10^{-4} \mathrm{~K}$, crosses). At $t \approx 2.4$, the system with $T=6 \times 10^{-4} \mathrm{~K}$ makes a transition from the metastable state to the stable state. At this temperature, the phase of one randomly chosen oscillator [horizontal coordinate 52, vertical coordinate $76,(0,0)$ being in the lower left corner] is shown in the inset. For small $t$, the oscillator is part of the metastable domain and moves correspondingly fast. At $t \sim 3.1$, the spreading boundary of the defect attains the oscillator and its motion is slowed down (and its direction reversed) to the much lower frequency of the stable state.

est solution of Eq. (2) and (ii) does the system ever go into a state corresponding to other solutions? We shall see that the answers to both questions are intimately connected.

In order to answer the second question, we start the system from a spatially homogeneous state with

$$
\phi_{i}(t)=4 K(t+\tau),
$$

with $-\tau \leq t \leq 0$ and $K=50 \omega_{0}, \omega_{0}=0.5$. Figure 2 shows that for small temperatures $\left(T=6 \times 10^{-4} \mathrm{~K}\right)$ the system stays for some time in a metastable state with a frequency $\Omega_{\max }$, which is the largest stable solution of Eq. (2), and then decays to a state with the lowest possible common frequency $\boldsymbol{\Omega}_{\min }$, which is again approximately given by Eq. (2). The color plates (Fig. 3) show that this instability is due to a spatial inhomogeneity in the phase distribution of the system which is caused by a thermal fluctuation and which increases in the course of time. Figure 2 shows that for higher temperatures the probability of phase slips increases, leading to a faster decay into a state with the lowest possible frequency, while for lower temperatures the occurrence of a spatial inhomogeneity is less probable and the system stays longer in the metastable state.

Numerical experiments indicate that the stable state is characterized by a phase distribution which varies slowly in space [see, e.g., Fig. 3(d)]. In this case, we can linear- ize the sine function in the interaction term of Eq. (1) The linearized equation $\dot{\phi}_{i}=\omega_{0}-K \sum_{j}\left[\phi_{i}(t)-\phi_{j}(t-\tau)\right]$ can be solved by Fourier transformation which leads to the averaged frequency $\langle\dot{\phi}\rangle=\omega_{0}(1+K n \tau)^{-1}$.

We have still to answer the question of why the smallest solution of Eq. (2) is globally stable and the higher solutions are not. The existence of metastable states which become destabilized by spatial phase fluctuations can be understood in the following model of two limitcycle oscillators with phases $\phi_{1}(t), \phi_{2}(t)$, which are coupled with time delay:

$$
\begin{aligned}
& \dot{\phi}_{1}=\omega_{1}-K \sin \left(\phi_{1}-\lambda \psi_{2}\right), \\
& \dot{\phi}_{2}=\omega_{2}-K \sin \left(\phi_{2}-\lambda \psi_{1}\right), \\
& \dot{\psi}_{1,2}=\phi_{1,2}-\lambda \psi_{1,2} .
\end{aligned}
$$

Since Eqs. (4)-(6) can be solved as

$$
\lambda \psi_{1,2}(t)=\frac{\int_{0}^{t} d \tau e^{-\lambda \tau} \phi_{1,2}(t-\tau)}{\int_{0}^{\infty} d \tau \epsilon^{-\lambda \tau}},
$$

we see that the variables $\lambda \psi_{1,2}(t)$ are the average over the delayed phase variables $\phi_{1,2}(t-\tau)$ with an exponential distribution of delays [15]. This modification of the original problem where we had only a single delay time allows us to visualize the existence and stability of metastable states in a simple two-dimensional potential.

For $\mathrm{x}=\left(x_{1}, x_{2}\right)$ with $x_{1}=\left(\phi_{1}+\lambda \psi_{1}-\phi_{2}-\lambda \psi_{2}\right) / 2$ and $x_{2}=\left(\phi_{1}-\lambda \psi_{1}+\phi_{2}-\lambda \psi_{2}\right) / 2$, Eqs. (4)-(6) reduce to

$$
\begin{aligned}
& \dot{\mathbf{x}}=-\nabla V(\mathbf{x}), \\
& \dot{\psi}_{1}-\dot{\psi}_{2}=2 x_{1}-2 \lambda\left(\psi_{1}-\psi_{2}\right), \\
& \dot{\psi}_{1}+\dot{\psi}_{2}=2 x_{2},
\end{aligned}
$$

with a potential

$V(\mathbf{x})=-x_{1} \Omega_{-}-K \cos x_{1} \cos x_{2}-x_{2} \Omega_{+}+\frac{1}{2} \lambda x_{2}^{2}$,

shown in Fig. 4 for $\Omega_{ \pm}=\left(\omega_{1} \pm \omega_{2}\right) / 2$. If the system is trapped in a local minimum $\left(x_{1}^{*}, x_{2}^{*}\right)$ of $V(\mathbf{x})$, the phase difference between the oscillators becomes $\phi_{1}(t)-\phi_{2}(t)$ $=x_{1}^{*}$, and the common frequency becomes $\dot{\phi}_{1}=\dot{\phi}_{2}=x_{2}^{*} \lambda$ [16]. Therefore the axes $x_{1}, x_{2}$ in Fig. 4 denote the phase difference and the common frequency of the delay coupled oscillators. For a spatially homogeneous situation $\left(x_{1}=0\right)$, the potential is a superposition of a shifted parabola with a periodic function and therefore has local minima whose depths increase with coupling $K$ and effective delay time $\tau_{0}=\lambda^{-1}$ [17]. In the presence of spatial inhomogeneities of the phases, i.e., for $\phi_{1}-\phi_{2} \neq 0$, the local minima become smaller and vanish for $\phi_{1}-\phi_{2}$ $=\pi u / 2 \quad(u= \pm 1, \pm 3, \ldots)$. Spatial inhomogeneities therefore allow the system to reach the state with the lowest frequency at the bottom of the potential without climbing over local maxima. This agrees qualitatively with our numerical observations for the many-oscillator system. 
(a)

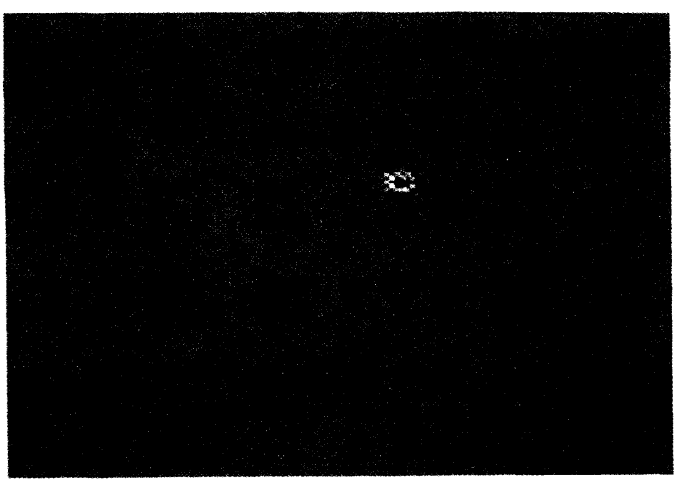

(c)

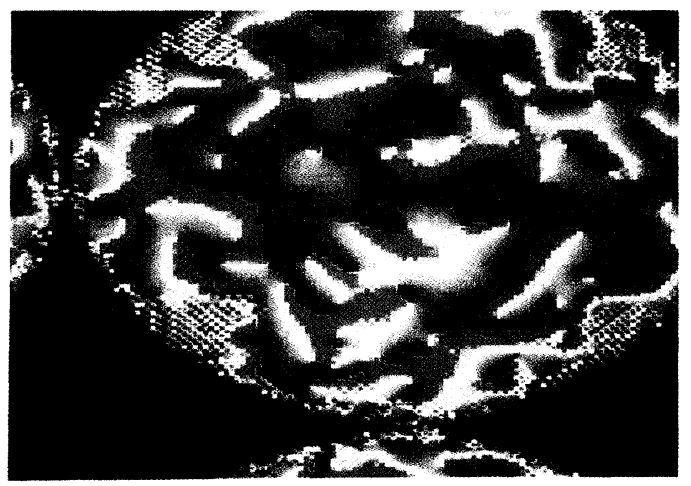

(b)

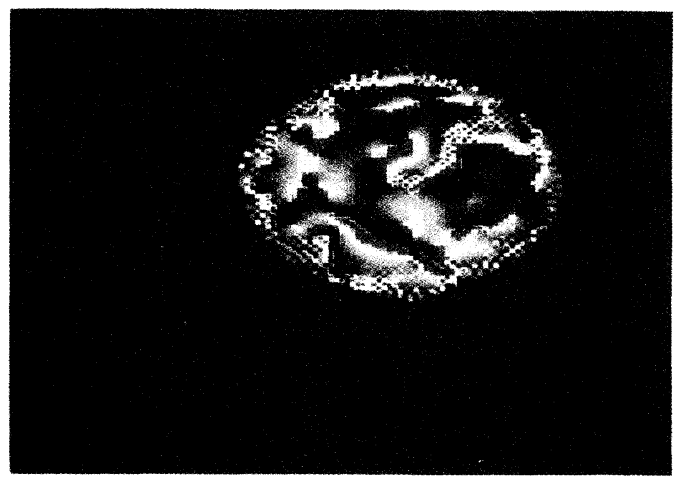

(d)

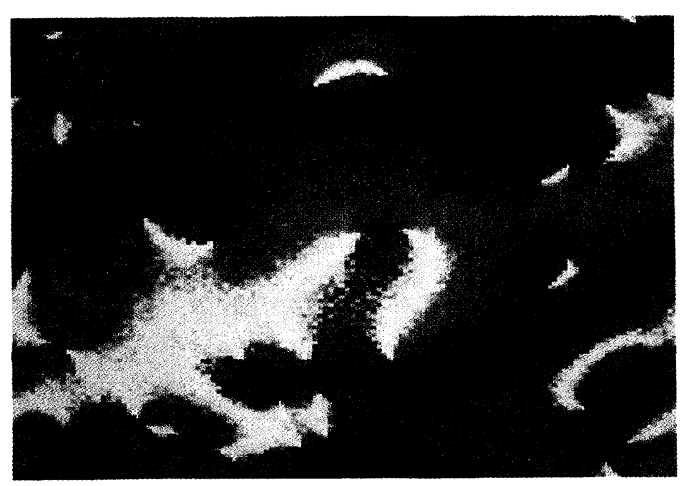

FIG. 3. Phases of $128 \times 128$ oscillators, using a color code where dark blue corresponds to $\phi=0$ and red to $\phi=2 \pi$, with rainbow colors for intermediate $\phi . T=6 \times 10^{-4} \mathrm{~K}, \omega_{0}=0.5$. (a) At time $t=2.4$, the system is in a quasihomogeneous state except in a small region where an inhomogeneity evolves. For earlier times, the system is homogeneous, except for small thermal fluctuations $(\delta \phi \lesssim 0.15 \times 2 \pi)$. In the next plates (b), (c), at times $t=3.0$ and 3.6, respectively, the rapid growth of the inhomogeneity is shown. A typical late state of the system is depicted in plate $(\mathrm{d})(t=8)$.

Finally, we would like to emphasize that the existence of metastable states and the drastic decrease of the synchronization frequency (which occurs even for small delay times if the coupling strength is large) should be observed in a wide range of dynamic systems. Our analysis has shown that these phenomena depend only on the generic periodicity of the interaction between the phases [17] and that they are relatively insensitive to the spectrum of intrinsic frequencies [15]. The existence and the functional form of the potential $V(x)$ depend on the details of the coupling between the oscillators [18], but the existence of the frequency depression does not. In the present work, we were able to reduce the many-body problem to the treatment of one-body or two-body systems. An understanding of the full many-body problem is still open and deserves further study. In general, we believe that the investigation of the nature of metastable states in dynamic systems with internal delay times which are abundant in nature is a promising direction of further research.

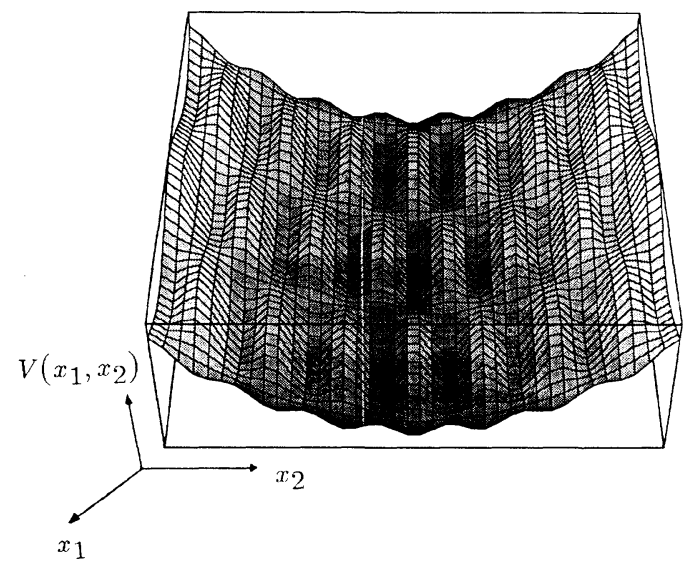

FIG. 4. The interaction potential, $V\left(x_{1}, x_{2}\right)$, for the two limit-cycle oscillator case [Eq. (11)]. The view is down the $x_{1}$ axis, showing the quadratic basin of attraction and the local minima. Note the lines where the potential is purely parabolic, without local minima. The parameters are $\Omega_{+}=\Omega_{-}=1.00$, $\lambda=50, K=2000, x_{1} \in[-5,5], x_{2} \in[-20,20]$. 
We are grateful to D. Ruderman for writing part of the Connection Machine code. We would like to thank B. Ermentrout and H. Sompolinsky for stimulating conversations and C. Koch for his continuing support and encouragement. Part of the computations have been performed on the Caltech/Argonne Connection Machine. E.N. is supported by the Swiss National Science Foundation through Grant No. 8220-25941. H.G.S. is supported by the Volkswagen Foundation, and D.M.K. by the Division of Biology and grants from the Air Force Office of Scientific Research and a NSF Presidential Young Investigator Award (both to C. Koch).

(a) Permanent address: Institut für Theoretische Physik, Universität Kiel, Olshausenstrasse 40, D-2300 Kiel 1, Germany.

(b) Present address: Department of Physics, Harvard University, Cambridge, MA 02138.

[1] J. Benford, H. Sze, W. Woo, and R. R. Smith, Phys. Rev. Lett. 62, 969 (1989); D. S. Fisher, Phys. Rev. B 31, 1396 (1985); S. H. Strogatz, C. M. Marcus, R. M. Westervelt, and R. E. Mirollo, Phys. Rev. Lett. 61, 2380 (1988); H. Daido, Phys. Rev. Lett. 61, 231 (1988).

[2] Y. Kuramoto and I. Nishikawa, J. Stat. Phys. 49, 569 (1987); H. Sakaguchi, S. Shinomoto, and Y. Kuramoto, Prog. Theor. Phys. 77, 1005 (1987).

[3] R. Loft and T. A. DeGrand, Phys. Rev. B 35, 8528 (1987).

[4] C. M. Gray, P. König, A. K. Engel, and W. Singer, Nature (London) 338, 334 (1989).

[5] A. T. Winfree, J. Theor. Biol. 16, 15 (1967); The Geometry of Biological Time (Springer-Verlag, New York, 1980).

[6] H. Sompolinsky, D. Golomb, and D. Kleinfeld, Phys. Rev. A 43, 6990 (1991).

[7] A. H. Cohen, P. J. Holmes, and R. H. Rand, J. Math.
Biol. 13, 345 (1982).

[8] H. G. Schuster and P. Wagner, Prog. Theor. Phys. 81, 939 (1989); H. G. Schuster and P. Wagner, Biol. Cybernetics 64, 77 (1990); 64, 83 (1990).

[9] R. T. Byerly and E. W. Kimball, Stability of Large Electric Power Systems (IEEE, New York, 1974).

[10] C. M. Marcus and R. H. Westervelt, Phys. Rev. A 39, 347 (1989).

[11] W. Freeman, Sci. Am. 264 (2), 78 (1991).

[12] S. H. Strogatz and R. E. Mirollo, J. Phys. A 21, L688 (1988); P. C. Matthews and S. H. Strogatz, Phys. Rev. Lett. 65, 1701 (1990).

[13] G. B. Ermentrout and N. Kopell, SIAM J. Appl. Math. 50, 125 (1990).

[14] E. Niebur, D. M. Kammen, and C. Koch, in Neural Information Processing Systems 3, edited by D. S. Touretzky (Morgan Kaufmann, San Mateo, 1991), p. 123; in Nonlinear Dynamics and Neuronal Networks, edited by W. Singer and H. G. Schuster (VCW Verlag, Weinheim, Germany, 1991), p. 173; E. Niebur, H. G. Schuster, D. M. Kammen, and C. Koch, Phys. Rev. A (to be published).

[15] The delayed phase variable $\phi(t-\tau)=\int d \tau^{\prime} \delta\left(\tau^{\prime}-\tau\right) \phi(t$ $\left.-\tau^{\prime}\right)$ is a special case where the distribution of delays is a $\delta$ function.

[16] At $x_{1}^{*}, x_{2}^{*}$, we have from Eq. (9) $\psi_{1}(t)-\psi_{2}(t)=x_{1}^{*} / \lambda$ $+\left[\psi_{1}(0)-\psi_{2}(0)-x_{1}^{*} / \lambda\right] e^{-2 \lambda i}$, i.e., $\lim _{t} \cdot \infty \psi_{1}(t)-\psi_{2}(t)$ $=x_{1}^{*} / \lambda$. This yields in the long-time limit, from Eq. (10), $\psi_{1,2}=\left(x_{1}^{*} \pm x_{1}^{*}\right) / 2 \lambda+x_{2}^{*} t$ and, from Eq. (6), $\phi_{1,2}(t)$ $=\left(x_{1}^{*} \pm x_{1}^{*}\right) / 2+x_{2}^{*}+\lambda x_{2}^{*} t$.

[17] In this case, both oscillators become identical for $\omega_{1}$ $=\omega_{2}=\omega$. The equations for the common phase reduce to $\dot{\phi}=\omega-\Gamma(\phi(t)-\lambda \psi(t)), \dot{\psi}=\phi-\lambda \psi$, where the interaction $\Gamma(x)=\Gamma(x+2 \pi n)$ with an integer $n$ can be any periodic function. The potential reduces to $V\left(x_{2}\right)$ $=-K \int_{0}^{x^{2}} \Gamma(x) d x-\Omega x_{2}+\frac{1}{2} \lambda x_{2}^{2}$, which shows that the metastable states can be traced back to this generic periodicity of the interaction between the phases.

[18] N. Kopell and G. B. Ermentrout, Commun. Pure Appl. Math. 39, 623 (1986). 
(a)

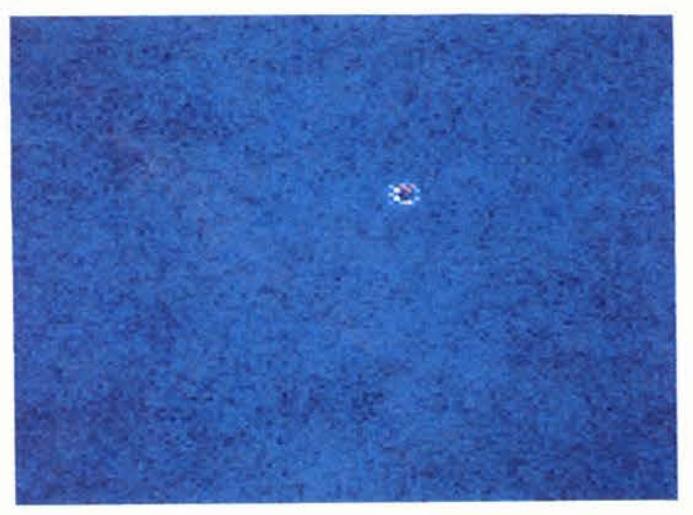

(c)

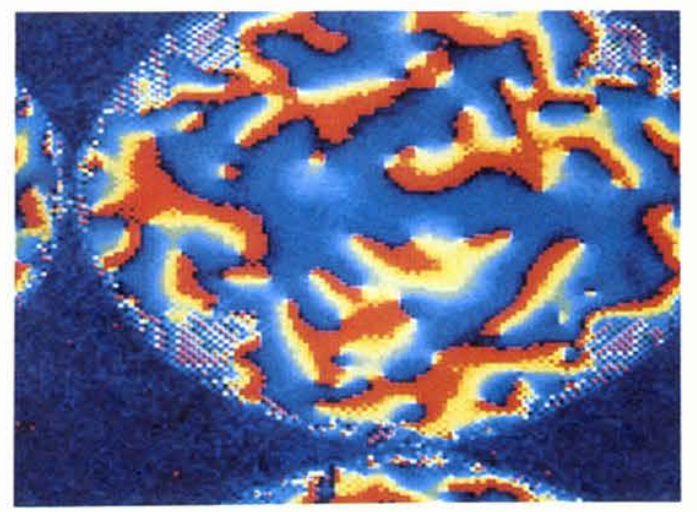

(b)

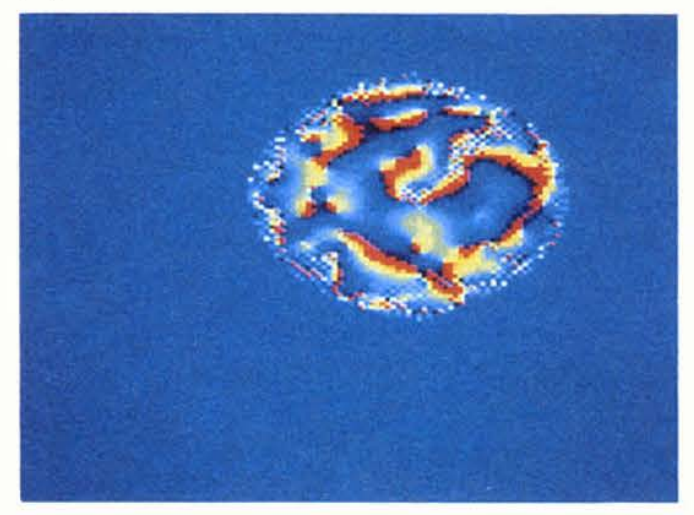

(d)

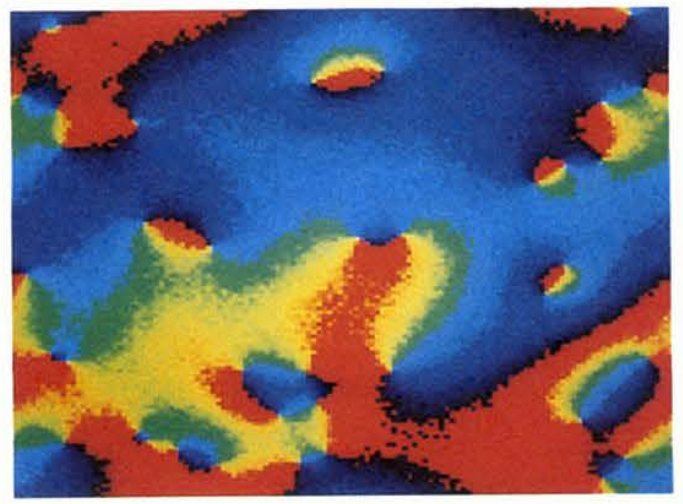

FIG. 3. Phases of $128 \times 128$ oscillators, using a color code where dark blue corresponds to $\phi=0$ and red to $\phi=2 \pi$, with rainbow colors for intermediate $\phi . T=6 \times 10^{-4} \mathrm{~K}, \omega_{0}=0.5$. (a) At time $t=2.4$, the system is in a quasihomogeneous state except in a small region where an inhomogeneity evolves. For earlier times, the system is homogeneous, except for small thermal fluctuations $(\delta \phi \lesssim 0.15 \times 2 \pi)$. In the next plates (b), (c), at times $t=3.0$ and 3.6, respectively, the rapid growth of the inhomogeneity is shown. A typical late state of the system is depicted in plate $(d)(t=8)$. 